\title{
Correction to: Non-commutative Calculus, Optimal Transport and Functional Inequalities in Dissipative Quantum Systems
}

\section{Eric A. Carlen ${ }^{1}$. Jan Maas ${ }^{2}$ \\ Published online: 18 November 2020 \\ (c) The Author(s) 2020 \\ Correction to: Journal of Statistical Physics (2020) 178:319-378 https://doi.org/10.1007/s10955-019-02434-w}

The claimed bound $\operatorname{Ric}(\mathcal{A}, \nabla, \tau) \geq \gamma$ in Theorem 10.6 in our paper [1] is unfortunately incorrect, as pointed out in [2]. A small modification of the proof shows that the weaker estimate $\operatorname{Ric}(\mathcal{A}, \nabla, \tau) \geq \frac{\gamma}{2}$ holds.

This bound can be obtained by replacing (10.5) by the following computation, using the scalar inequalities $\partial_{1} \Lambda(a, b), \partial_{2} \Lambda(a, b) \geq 0$ for $a, b>0$ :

$$
\begin{aligned}
\operatorname{Hess}_{\mathscr{K}} \operatorname{Ent}(\rho)[A, A] & =-\tau\left[(\nabla \mathscr{L} A)^{*} \widehat{\rho} \# \nabla A\right]+\tau\left[(\nabla A)^{*} \mathcal{N}_{\rho, \mathscr{L}^{\dagger} \rho}^{(\eta)} \#(\nabla A)\right] \\
& =\frac{\gamma}{2} \tau\left[(\nabla A)^{*}\left(\Lambda+\partial_{1} \Lambda+\partial_{2} \Lambda\right)(\rho, \rho) \#(\nabla A)\right] \\
& \geq \frac{\gamma}{2} \tau\left[(\nabla A)^{*} \Lambda(\rho, \rho) \#(\nabla A)\right] \\
& =\frac{\gamma}{2} \tau\left[(\nabla A)^{*} \widehat{\rho} \# \nabla A\right]=\frac{\gamma}{2}\left\langle\mathscr{K}_{\rho} A, A\right\rangle_{L^{2}(\tau)} .
\end{aligned}
$$

On the matrix algebra $\mathbb{M}_{n}(\mathbb{C})$, it is possible to improve the curvature bound $\frac{\gamma}{2}$ to $\frac{\gamma}{2}\left(1+\frac{1}{n}\right)$, using the scalar inequality $\partial_{1} \Lambda(a, b)+\partial_{2} \Lambda(a, b) \geq 1 \geq \frac{1}{n} \Lambda(a, b)$ for $0<a, b \leq n$.

We thank Michael Brannan and Li Gao and Marius Junge for pointing out the error, and Melchior Wirth and Haonan Zhang for useful discussions.

Acknowledgements E.C. gratefully acknowledges support through NSF grant DMS-174625. J.M. gratefully acknowledges support by the European Research Council (ERC) under the European Union's Horizon 2020 research and innovation programme (grant agreement No 716117), and by the Austrian Science Fund (FWF), Project SFB F65.

The original article can be found online at https://doi.org/10.1007/s10955-019-02434-w.

$凶 \quad$ Jan Maas

jan.maas@ist.ac.at

Eric A. Carlen

carlen@math.rutgers.edu

1 Department of Mathematics, Hill Center, Rutgers University, 110 Frelinghuysen Road, Piscataway, NJ 08854-8019, USA

2 Institute of Science and Technology Austria (IST Austria), Am Campus 1, 3400 Klosterneuburg, Austria 


\section{References}

1. Carlen, E.A., Maas, J.: Non-commutative calculus, optimal transport and functional inequalities in dissipative quantum systems. J. Stat. Phys. 178(2), 319-378 (2020)

2. Brannan, M., Gao, L., Junge, M.: Complete logarithmic Sobolev inequalities via Ricci curvature bounded below. arXiv:2007.06138v1 (2020)

Publisher's Note Springer Nature remains neutral with regard to jurisdictional claims in published maps and institutional affiliations. 